\title{
Detection of Bovine IgG Isotypes in a PPA-ELISA for Johne's Disease Diagnosis in Infected Herds
}

\author{
Bárbara Fernández, ${ }^{1}$ Liliana Rosa Gilardoni, ${ }^{1}$ Ana Jolly,, ${ }^{1}$ Silvia Beatriz Colavecchia, ${ }^{1}$ \\ Fernando Alberto Paolicchi, ${ }^{2,3}$ and Silvia Leonor Mundo ${ }^{1}$ \\ ${ }^{1}$ Cátedra de Inmunología, Facultad de Ciencias Veterinarias, Universidad de Buenos Aires (UBA), Chorroarín 280, \\ Ciudad Autónoma de Buenos Aires C1427CWO, Argentina \\ ${ }^{2}$ Laboratorio de Bacteriología, Estación Experimental Agropecuaria Balcarce, Instituto Nacional de Tecnología Agropecuaria (INTA), \\ Ruta 226, Km 73.5, Provincia de Buenos Aires, Balcarce B7620BEN, Argentina \\ ${ }^{3}$ Departamento de Producción Animal, Facultad de Ciencias Agrarias, Universidad Nacional de Mar del Plata (UNMdP), \\ Ruta 226, Km 73.5, Provincia de Buenos Aires, Balcarce B7620BEN, Argentina
}

Correspondence should be addressed to Silvia Leonor Mundo, smundo@fvet.uba.ar

Received 6 February 2012; Accepted 6 May 2012

Academic Editor: Michael D. Welsh

Copyright (c) 2012 Bárbara Fernández et al. This is an open access article distributed under the Creative Commons Attribution License, which permits unrestricted use, distribution, and reproduction in any medium, provided the original work is properly cited.

\begin{abstract}
Johne's Disease or Paratuberculosis is a chronic granulomatous enteritis disease affecting ruminants. Detection of subclinically infected animals is difficult, hampering the control of this disease. The aim of this work was to evaluate the performance of detection of IgG isotypes in a PPA-ELISA to improve the recognition of cattle naturally infected with Map in different stages. A total of 108 animals from Tuberculosis-free herds were grouped as follows: exposed $(n=30)$, subclinically infected ( $n=26)$, clinically infected $(n=14)$, and healthy controls $(n=38)$. Receiver-operating characteristic (ROC) curves of isotypes/PPA-ELISAs were constructed and areas under the curves were compared to evaluate the performance of each test. Our study demonstrated that the conventional PPA-ELISA (detecting IgG) is the best to identify clinically infected animals with high sensitivity (92.9\%) and specificity (100\%). Meanwhile, IgG2/PPA-ELISA improved the number of subclinically infected cattle detected as compared with conventional IgG/PPA-ELISA (53.8 versus $23.1 \%$ ). In addition, it had the maximum sensitivity $(65.0 \%$, taking into account all Map-infected cattle). In conclusion, the combination of IgG and IgG2/PPA-ELISAs may improve the identification of Mapinfected cattle in different stages of disease. The usefulness of IgG2 detection in serological tests for Johne's Disease diagnosis should be further evaluated.
\end{abstract}

\section{Introduction}

Johne's Disease (JD) or Paratuberculosis is a chronic granulomatous enteritis disease affecting ruminants $[1,2]$. It is caused by Mycobacterium avium subsp. paratuberculosis (Map) and leads to major economic losses in the dairy industry worldwide [3]. Map has been implicated as a possible cause of Crohn's disease, which is a chronic granulomatous ileocolitis in humans. However, its role in this pathology remains controversial [4-6].

Calves are the most susceptible category during the first months of life and become infected through ingestion of Map-contaminated colostrum, milk, or feces $[2,7]$. Fetal transmission is also possible when dams are infected with Map $[7,8]$.
During initial infection, the immune response is predominated by a cell-mediated immune profile (Th1). Subclinically infected animals are generally low Map fecal shedders and have undetectable levels of Map-specific serum antibodies and increasing specific gamma interferon (IFN- $\gamma$ ) responses [9]. After a long incubation period (years), a proportion of infected animals develop to a clinical stage, which is characterized by chronic diarrhea, protein-losing enteropathy, cachexia, and eventual death. In addition, increases in bacterial shedding in feces and serum antibody titers have been described in this stage of JD, suggesting a shift of the immune response to a humoral profile (Th2) $[1,10,11]$. The humoral immune response against mycobacterial infections has been considered nonprotective [1, 2]. However, it has been demonstrated that antibodies have an active role in 
Map infection in vitro. Map immune sera or purified specific antibodies enhance bacterial interaction with macrophages, improve the activation of the nuclear factor NF-kB in infected cells, and affect Map intracellular viability [12-14].

The control of JD has been difficult for several reasons. Fecal culture on conventional solid media is expensive, laborious and slow (requiring 6 months for assay ending), and has low sensitivity [15-17]. Detection of cellular immune response by either the skin test or IFN- $\gamma$ production is useful for early diagnosis of infection, but these assays have high variability and low specificity $[18,19]$. Vaccines have been demonstrated to decrease the amount of Map shedding, to prevent the development of the clinical stage and to reduce the impact on milk production. However, they do not prevent infection and shedding of the bacteria and interfere with Tuberculosis and JD diagnosis [20].

Although conventional ELISA (detecting IgG) has low sensitivity during the subclinical stage of the infection, it is the test most used for JD control due to its low-cost, highthroughput, standardized protocols, and correlation with Map fecal shedding levels [21-23]. Various antigens of Map have been studied, including protoplasmic antigen (PPA), lipoarabinomannan (LAM), p34 protein carboxy-terminal (P34-cx), purified protein derivative (PPDp), and heat shock proteins (Hsp), of which PPA is the one most used for diagnosis [21-23]. Production of Map-specific isotypes switches during the course of the disease $[10,24,25]$ with Th1 responses being related to IgM and IgG2, and Th2 responses being related to IgG1 and IgA in cattle [26]. In the same way, high levels of specific IgG1 against several antigens have been detected in sera from Map-infected cattle at a clinical stage of the disease $[13,14,24,25]$. In a previous study, we have shown increases in the levels of Map-specific IgG2 in cattle at both the subclinical and clinical stages of JD [25].

The aim of this work was to evaluate the performance of detection of IgG isotypes in a PPA-ELISA to improve the recognition of cattle naturally infected with Map in different stages of the disease.

\section{Materials and Methods}

2.1. Animals. Sera from 108 Holstein-Frisian bovines from Tuberculosis-free accredited dairy herds from the Pampas region of Argentina were used to assess the performance of IgG, IgG1, and IgG2/PPA-ELISAs.

JD diagnosis was achieved as previously described [25]. Briefly, we examined animals for clinical signs of disease and for Map presence in milk and fecal-isolated colonies by PCR identification of the IS900 fragment. Milk samples were concentrated by Map-specific immunomagnetic beads (NEB, New England Biolabs, Ipswich, MA, USA) [27, 28]. Fecal cultures were carried out in Herrold egg yolk medium with mycobactin J (Allied Monitor Inc., Fayette, MO, USA) and pyruvate (Sigma-Aldrich Corp., St. Louis, MO, USA).

Animals were grouped as follows:

(i) exposed (E, $n=30)$ : from Map-infected herds, without clinical signs of JD and negative to IS900-PCR (from feces and milk); (ii) subclinically infected (SC, $n=26$ ): from Map-infected herds, without clinical signs of JD and positive to IS900-PCR (from feces, milk, or both);

(iii) clinically infected (C, $n=14$ ): from Map-infected herds with chronic diarrhea and positive to IS900PCR (from feces, milk, or both);

(iv) healthy control ( $\mathrm{Hc}, n=38)$ : from Map-free herds and negative to IS900-PCR (from feces and milk).

2.2. ELISAs. IgG, IgG1, and IgG2/PPA-ELISAs were evaluated using sera from the 108 bovines. Cross-reactive antibodies were preadsorbed with Mycobacterium phlei [29], which had been grown at $37^{\circ} \mathrm{C}$ in Middlebrook $7 \mathrm{H} 9$ broth (DifcoTM, BD biosciences, Franklin Lakes, NJ, and USA) containing $10 \%$ albumin-dextrose-sodium chloride and then heat-inactivated at $85^{\circ} \mathrm{C}$ for 30 minutes. For preadsorption, sera diluted 1:5 with PBS containing heat-inactivated Mycobacterium phlei (optical density (OD) at $600 \mathrm{~nm}$ of 1) were incubated at $37^{\circ} \mathrm{C}$ for $1 \mathrm{~h}$ with shaking, and then at $4{ }^{\circ} \mathrm{C}$ for $16 \mathrm{~h}$.

Flat-bottomed 96-well polystyrene plates were coated $\left(4^{\circ} \mathrm{C}, 16 \mathrm{~h}\right.$ ) with $2 \mu \mathrm{g} /$ well of PPA (Allied Monitor Inc.) in $50 \mu \mathrm{L}$ of $0.05 \mathrm{M}$ sodium carbonate buffer $\mathrm{pH}$ 9.6. The plates were washed three times with rinsing buffer $(0.05 \%$ Tween 20 in PBS) and blocked with 10\% skimmed milk in PBS. All subsequent incubations were performed at $37^{\circ} \mathrm{C}$ for $1 \mathrm{~h}$ and after each incubation, plates were washed three times with rinsing buffer. A volume of $50 \mu \mathrm{L}$ of preadsorbed sera at a final dilution of 1:5 (for IgG2 analyses) or 1:100 (for IgG and IgG1 analyses) in 5\% skimmed milk in PBS was added. The antibodies used were: HRP-conjugated goat anti-bovine IgG (KPL, Kirkegaard \& Perry Laboratories Inc., Gaithsburg, MD, USA), HRP-conjugated sheep anti-bovine IgG1 (Bethyl Laboratories Inc., Montgomery, TX, USA), and mouse monoclonal anti-bovine IgG2 (Sigma-Aldrich Co.) followed by HRP-conjugated goat anti-mouse IgG (KPL). Plates were developed using ortho-phenylendiamine dihydrochloride (OPD, Sigma-Aldrich Co.) in citrate buffer (Sigma-Aldrich Co.) and read in an OpsysMR spectrophotometer (Dynex Technologies, Chantilly, VA, USA). Results are expressed as mean OD values at $490 \mathrm{~nm}$.

2.3. Data Analysis. All experiments were conducted in duplicate or triplicate and repeated at least twice.

STATISTIX 8.0 (Analytical software, Tallahassee, USA) was used to analyze data of the humoral immune response against PPA. The logarithms of the mean OD values obtained were compared between groups. The levels of IgG and IgG2 were studied with ANOVA followed by Tukey's test, whereas those of IgG1 were analyzed with the Kruskal-Wallis test followed by pairwise comparisons.

CurvMedCalc Software version 12 (Mariakerke, Belgium) was used to evaluate the power of the $\operatorname{IgG}, \operatorname{IgG} 1$, and IgG2/PPA-ELISAs and to build the Receiver-operating characteristic (ROC) curves of infected cattle. The sensitivity of each test was estimated as \% of infected cattle (subclinically infected, clinically infected, or both) testing positive at the cut-off chosen. The specificity of each test 


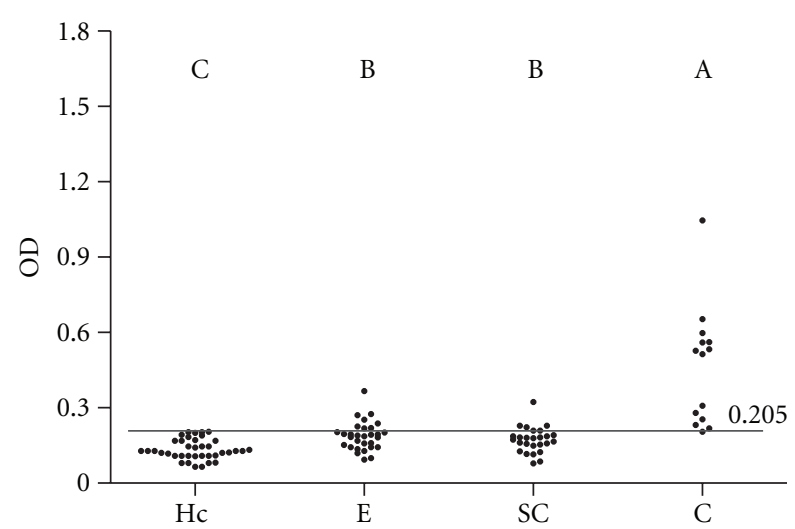

(a)

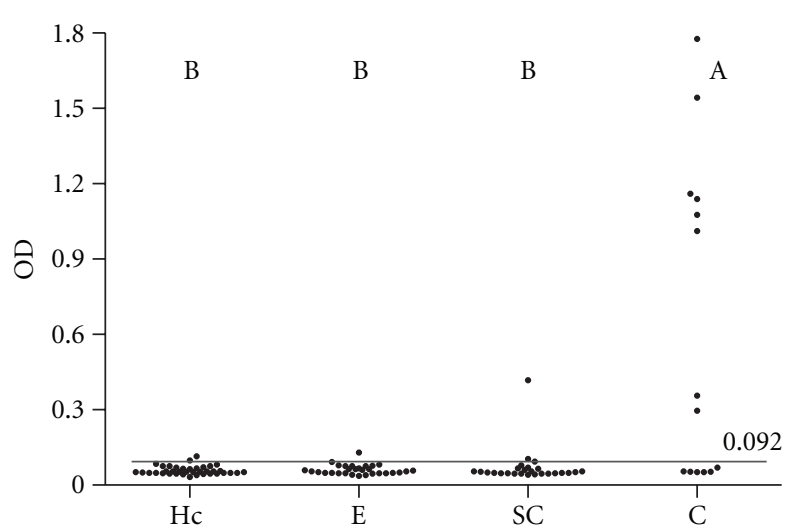

(b)

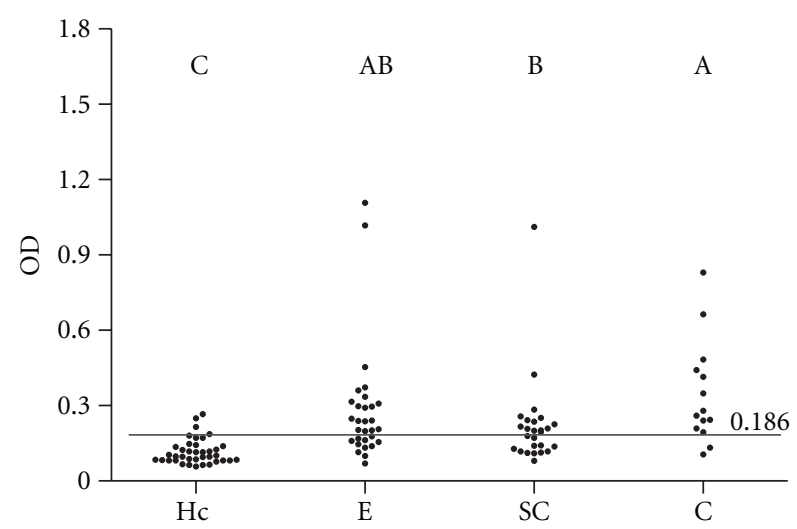

(c)

FIGURE 1: Results of isotypes/PPA-ELISAs. Dotplots of IgG (a), IgG1 (b), and IgG2 (c) PPA-ELISAs. Antibody responses are plotted as mean optical density (OD); lines and numbers (over lines) represent cut-off points. Letters indicate a significant difference $(P<0.05)$ between groups. Groups: healthy controls ( $\mathrm{Hc}, n=38)$, exposed $(\mathrm{E}, n=30)$, subclinically infected (SC, $n=26)$, and clinically infected (C, $n=14)$.

was calculated as \% of cattle from the healthy control group testing negative at the cut-off chosen. ROC curves for IgG, IgG1 and IgG2/PPA-ELISAs of subclinically and clinically infected cattle were constructed as plots of sensitivity versus 100 minus specificity for each possible cut-off $[30,31]$. Different methods were applied to assess the cut-off points of each ELISA. The mean OD values of healthy control group \pm 2 standard deviation and the ROC curves of infected cattle were analyzed. Cut-off points were selected from the ROC curves in order to obtain the highest sensitivity for subclinically infected cattle with a specificity of at least $90 \%$. The area under each ROC curve (AUC) was estimated and AUCs were compared using the method described by DeLong et al [32].

The level of significance was set at a $P$ value $<0.05$.

\section{Results}

Results of the isotypes/PPA-ELISAs in sera from healthy control, exposed, subclinically infected, and clinically infected cattle are shown in Figure 1 and Table 1. PPA-specific IgG was significantly increased in sera from all groups of Mapinfected herds (exposed, subclinically infected, and clinically infected) compared with the healthy control group. In addition, the clinically infected group showed the highest values detected. When the groups were evaluated by the IgG1/PPA-ELISA, only the clinically infected group showed high levels of this isotype. Meanwhile, the levels of specific IgG2 were significantly increased in all groups from Mapinfected herds $(P<0.05)$.

The ROC curves of the IgG, IgG1, and IgG2/PPA-ELISAs for the subclinically and clinically infected groups are shown in Figure 2. As expected, the AUCs were higher for the clinically infected group than for the subclinically infected one (Table 2).

The IgG/PPA-ELISA showed the highest specificity $(100 \%)$ and sensitivity for clinically infected cattle $(92.9 \%$, Table 3). However, this test detected as positive only $6 / 26$ of the subclinically infected animals and 8/30 of the exposed animals (Table 1 ).

The IgG1/PPA-ELISA demonstrated low performance and low sensitivity (27.5\% of Map-infected cattle (subclinically and clinically infected), Figure 2, Tables 2 and 3 ).

The IgG2/PPA-ELISA showed $92.1 \%$ of specificity and the best performance for the subclinically infected group $(\mathrm{AUC}=0.812)$ as compared with the IgG/PPA-ELISA (AUC 


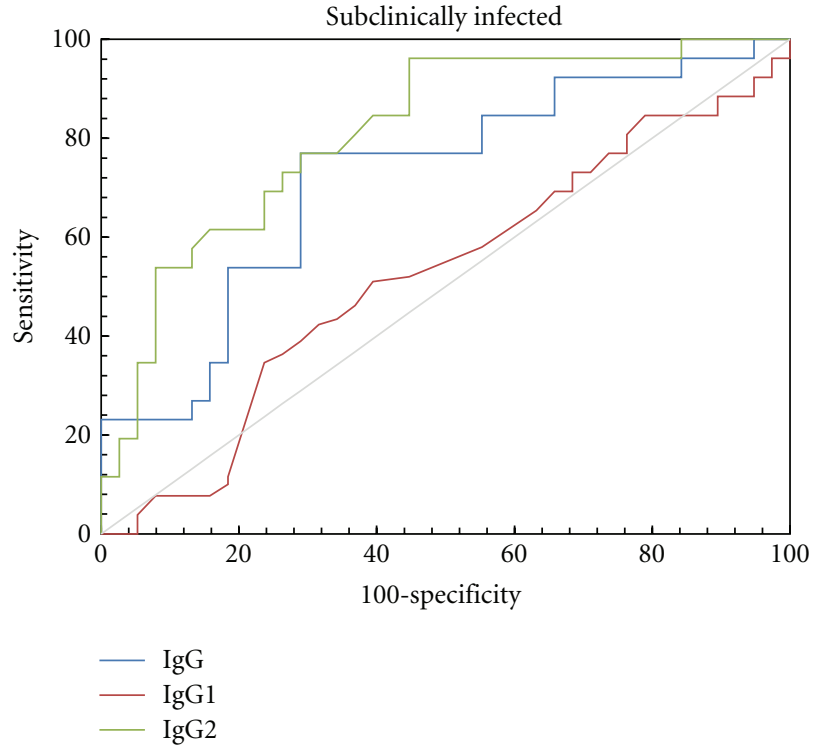

(a)

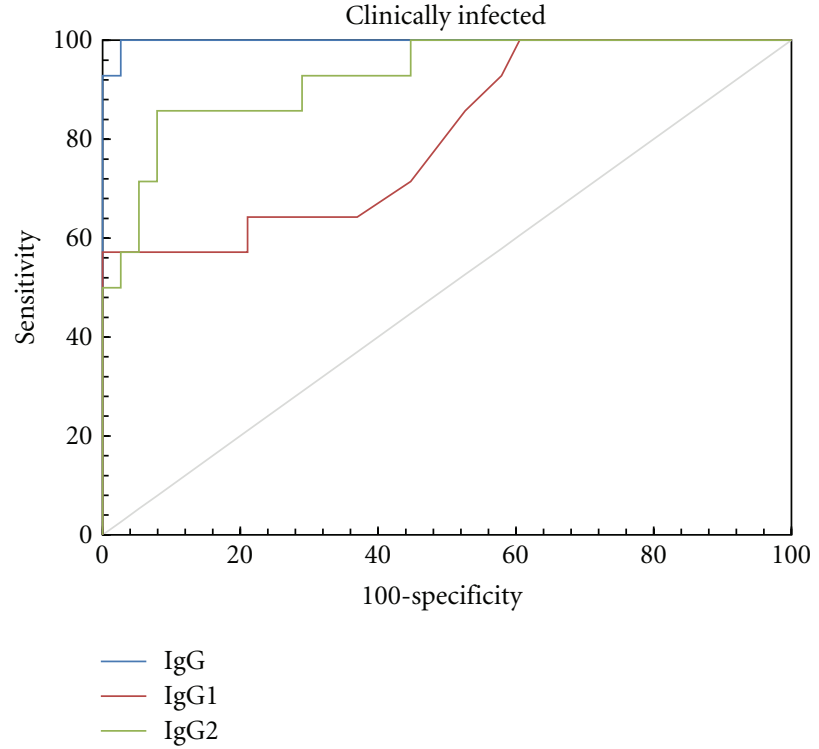

(b)

Figure 2: Performances of isotypes/PPA-ELISAs for subclinically infected (a) and clinically infected cattle (b). Receiver-operating characteristic (ROC) curves for IgG, IgG1, and IgG2/PPA-ELISAs.

TABle 1: Percentages of positivity of isotypes/PPA-ELISAs.

\begin{tabular}{lccc}
\hline Groups & IgG/PPA-ELISA & IgG1/PPA-ELISA & IgG2/PPA-ELISA \\
\hline Hc & $0.0 \%(0 / 38)$ & $5.3 \%(2 / 38)$ & $7.9 \%(3 / 38)$ \\
E & $26.7 \%(8 / 30)$ & $3.3 \%(1 / 30)$ & $63.3 \%(19 / 30)$ \\
SC & $23.1 \%(6 / 26)$ & $11.5 \%(3 / 26)$ & $53.8 \%(14 / 26)$ \\
C & $92.9 \%(13 / 14)$ & $57.1 \%(8 / 14)$ & $85.7 \%(12 / 14)$ \\
\hline
\end{tabular}

Numbers of positive animals are shown between brackets. Groups: healthy controls (Hc, $n=38$ ), exposed (E, $n=30$ ), subclinically infected (SC, $n=$ $26)$, and clinically infected (C, $n=14)$.

$=0.719)$ and IgG1/PPA-ELISA (AUC = 0.526), detecting $53.8 \%$ of the subclinically infected animals and $63.3 \%$ of the exposed animals (Figure 2 and Tables 1-3). In addition, the IgG2/PPA-ELISA had the maximum sensitivity $(65.0 \%$, taking into account all Map-infected cattle) and was able to detect 26/40 of Map-infected cattle. In contrast, only 19/40 were identified by the IgG/PPA-ELISA.

\section{Discussion}

The response of isotypes in Map-infected cattle has been previously studied $[10,13,14,24,25]$. We have described Map-specific isotypes detecting high levels of IgG2 in sera from Map-infected cattle at both the subclinical and clinical stages of the disease $[13,25]$. Taking into account that PPA is the Map antigen most widely used [13, 22, 33], in the present work, we developed isotypes/PPA-ELISAs to evaluate their application in diagnosis of JD in cattle.

It has been described that Map-infected animals in the clinical stage are high shedders of bacteria in feces, and thus have the greatest potential to transmit Map to other animals of the herd $[7,34]$. Meanwhile, subclinically infected cattle usually shed lower levels of Map and they are the largest part of the Map-infected herds, so detection of these animals is considered of great importance for JD control [35].

In this work, we detected an increase in the level of PPAspecific IgG in sera from clinically infected animals. Similar responses against other Map antigens have been previously reported $[10,13,25]$. We also detected increases in the levels of specific IgG in the subclinically infected group, in contrast to our previous study using Map-whole bacteria as antigen [25]. The IgG/PPA-ELISA demonstrated a perfect specificity $(100 \%)$; this is in accordance with published studies that have described specificities from 94 to $100 \%[21,36]$.

Although specific IgG1 against Map-antigens has been described as characteristic of clinically infected animals [13, $14,24,25]$, in the present study the detection of PPA-specific IgG1 did not improve the diagnosis in this stage of disease.

Interestingly, the IgG2/PPA-ELISA allowed detecting the majority of subclinically and clinically infected animals, confirming our preliminary studies $[13,25]$.

Although sera were preadsorbed, three animals of the healthy control group showed OD values higher than the cutoff of the IgG2/PPA-ELISA (Figure 1 and Table 1 ). This could be related to the lower specificity (92.1\%).

Our study demonstrates that the IgG/PPA-ELISA is the best to identify clinically infected animals, with high sensitivity and specificity, in accordance with the accepted statement that conventional ELISAs mostly identify this category of infected cattle $[21,23]$.

On the other hand, our IgG2/PPA-ELISA improved the number of subclinically infected cattle detected as compared with conventional IgG/PPA-ELISA (53.8 versus $23.1 \%$ ), maintaining high levels of specificity. Nevertheless, this sensitivity is slightly lower than that reported by Paolicchi [33] using an IgG/PPA-ELISA, although this could be related to the number of animals included (26 versus 8 animals). 
TABLE 2: Performances of isotypes/PPA-ELISAs for subclinically infected and clinically infected cattle.

\begin{tabular}{|c|c|c|c|c|c|c|c|}
\hline \multicolumn{4}{|c|}{ Subclinically infected } & \multicolumn{4}{|c|}{ Clinically infected } \\
\hline \multirow{2}{*}{$\begin{array}{l}\text { PPA } \\
\text { ELISA } \\
\text { IgG }\end{array}$} & \multirow{2}{*}{$\begin{array}{c}\text { AUCs } \\
0.719\end{array}$} & \multicolumn{2}{|c|}{ Comparison of AUCs } & \multirow{2}{*}{$\begin{array}{c}\text { PPA } \\
\text { ELISA } \\
\text { IgG }\end{array}$} & \multirow{2}{*}{$\begin{array}{c}\text { AUCs } \\
0.998\end{array}$} & \multicolumn{2}{|c|}{ Comparison of AUCs } \\
\hline & & IgG $\sim \operatorname{IgG1}$ & $P=0.0858$ & & & IgG $\sim$ IgG1 & $P=0.0057$ \\
\hline IgG1 & 0.526 & $\operatorname{IgG} \sim \operatorname{IgG} 2$ & $P=0.2402$ & IgG1 & 0.805 & $\operatorname{IgG} \sim \operatorname{IgG} 2$ & $P=0.0721$ \\
\hline IgG2 & 0.812 & $\operatorname{IgG} 1 \sim \operatorname{IgG} 2$ & $P=0.0048$ & IgG2 & 0.927 & $\mathrm{IgG} 1 \sim \mathrm{IgG} 2$ & $P=0.0913$ \\
\hline
\end{tabular}

Estimated area under the curve (AUC) of each test and pairwise statistical analysis.

TABLE 3: Specificity and sensitivity of isotypes/PPA-ELISAs.

\begin{tabular}{|c|c|c|c|c|}
\hline \multirow{2}{*}{$\begin{array}{l}\text { PPA } \\
\text { ELISA }\end{array}$} & \multirow{2}{*}{ Specificity } & \multicolumn{3}{|c|}{ Sensitivity } \\
\hline & & Subclinically & Clinically & Total \\
\hline IgG & $100.0 \%$ & $23.1 \%$ & $92.9 \%$ & $47.5 \%$ \\
\hline IgG1 & $94.7 \%$ & $11.5 \%$ & $57.1 \%$ & $27.5 \%$ \\
\hline IgG2 & $92.1 \%$ & $53.8 \%$ & $85.7 \%$ & $65.0 \%$ \\
\hline
\end{tabular}

The specificity of isotypes/PPA-ELISAs was calculated as $\%$ of cattle from the healthy control group testing negative. The sensitivity of each test was estimated as \% of infected cattle (subclinically infected, clinically infected, or both) testing positive.

The sensitivity of fecal culture has been reported to be too low to define absence of Map infection for animals residing in known infected herds [21]. In fact, Map-infected cattle in the early stage may shed bacteria under detectable levels using current methods, including culture and PCR [35]. In the same way, Nielsen [37] has recently highlighted the importance of the study of Map-infected shedder and nonshedder animals to evaluate an immune-based diagnostic test. Thus, in the present work, we incorporated a group of exposed animals from Map-infected herds, excluding them from the specificity and sensitivity analysis. In this group, the use of IgG2/PPA-ELISA allowed detection of more positive animals than the other isotypes evaluated (63.3 versus 26.7 or 3.3\%). Using a IgG/PPA-ELISA test, Huda et al. detected $11 \%$ of exposed animals as positive [31].

New antigens have been proposed to increase the sensitivity of JD diagnosis by IgG/ELISA [34, 36, 38]. Thus, it could be interesting to evaluate those antigens in an IgG2/ELISA.

In conclusion, our results show that IgG2/PPA-ELISA improves detection of subclinically Map-infected cattle or herds with animals in all stages of JD and in combination with IgG/PPA-ELISA may improve differentiation of clinical stages of disease. More studies should be conducted to better approach the utility of the IgG2/PPA-ELISA, in which, naturally and experimentally infected cattle should be included and the infection status should be supported by histopathological examination and culture of tissues. In addition, the usefulness of IgG2 detection in serological tests for Johne's Disease diagnosis should be further evaluated.

\section{Acknowledgments}

The authors would like to thank Claudia Morsella for her valuable technical assistance, and VMD Soledad Barandiaran and Maria Laura Fortuny for their helpful collaboration in field sampling. This work was supported by Grants from the Agencia Nacional de Promoción Científica y Técnica (BID PICT 2010-2672) and from Universidad de Buenos Aires (UBA-SeCyT 20020100100912).

\section{References}

[1] R. J. Chiodini, "Immunology: resistance to paratuberculosis," The Veterinary Clinics of North America. Food Animal Practice, vol. 12, no. 2, pp. 313-343, 1996.

[2] N. B. Harris and R. G. Barletta, "Mycobacterium avium subsp. paratuberculosis in veterinary medicine," Clinical Microbiology Reviews, vol. 14, no. 3, pp. 489-512, 2001.

[3] J. E. Lombard, "Epidemiology and economics of paratuberculosis," The Veterinary Clinics of North America. Food Animal Practice, vol. 27, no. 3, pp. 525-535, 2011.

[4] S. Singh and G. Krishnamoorthy, "Mycobacterium avium subspecies paratuberculosis and Crohn's Regional Ileitis: how strong is association?" Journal of Laboratory Physicians, vol. 3, no. 2, pp. 69-74, 2011.

[5] C. D. William and S. A. Madsen-Bouterse, "Crohn's disease and Mycobacterium avium subsp. paratuberculosis: the need for a study is long overdue," Veterinary Immunology and Imunopathology, vol. 145, no. 1-2, pp. 1-6, 2012.

[6] R. J. Chiodini, W. M. Chamberlin, J. Sarosiek, and R. W. McCallum, "Crohn's disease and the mycobacterioses: a quarter century later. Causation or simple association?" Critical Reviews in Microbiology, vol. 38, no. 1, pp. 52-93, 2012.

[7] R. W. Sweeney, "Transmission of paratuberculosis," The Veterinary Clinics of North America. Food Animal Practice, vol. 12, no. 2, pp. 305-312, 1996.

[8] R. J. Whittington and P. A. Windsor, "In utero infection of cattle with Mycobacterium avium subsp. paratuberculosis: a critical review and meta-analysis," Veterinary Journal, vol. 179, no. 1, pp. 60-69, 2009.

[9] J. R. Stabel, "Production of $\gamma$-interferon by peripheral blood mononuclear cells: an important diagnostic tool for detection of subclinical paratuberculosis," Journal of Veterinary Diagnostic Investigation, vol. 8, no. 3, pp. 345-350, 1996.

[10] B. Abbas and H. P. Riemann, "IgG, IgM and IgA in the serum of cattle naturally infected with Mycobacterium paratuberculosis," Comparative Immunology, Microbiology and Infectious Diseases, vol. 11, no. 3-4, pp. 171-175, 1988.

[11] J. R. Stabel, "Transitions in immune responses to Mycobacterium paratuberculosis," Veterinary Microbiology, vol. 77, no. 3-4, pp. 465-473, 2000.

[12] J. Hostetter, R. Kagan, and E. Steadham, "Opsonization effects on Mycobacterium avium subsp. paratuberculosis macrophage interactions," Clinical and Diagnostic Laboratory Immunology, vol. 12, no. 6, pp. 793-796, 2005. 
[13] S. L. Mundo, A. M. Fontanals, M. García et al., "Bovine IgG1 antibodies against Mycobacterium avium subsp. paratuberculosis protein $\mathrm{p} 34-\mathrm{cx}$ improve association of bacteria and macrophages," Veterinary Research, vol. 39, no. 1, pp. 1-12, 2008.

[14] A. Jolly, S. B. Colavecchia, B. Fernández, E. Fernández, and S. L. Mundo, "Antibodies induced by lipoarabinomannan in bovines: characterization and effects on the interaction between Mycobacterium avium subsp. paratuberculosis and macrophages in vitro," Veterinary Medicine International, vol. 2011, Article ID 258479, 8 pages, 2011.

[15] J. B. Jørgensen, "An improved medium for culture of Mycobacterium paratuberculosis from bovine faeces," Acta Veterinaria Scandinavica, vol. 23, no. 3, pp. 325-335, 1982.

[16] M. T. Collins, "Diagnosis of paratuberculosis," The Veterinary Clinics of North America. Food Animal Practice, vol. 12, no. 2, pp. 357-371, 1996.

[17] L. de Juan, J. Álvarez, B. Romero et al., "Comparison of four different culture media for isolation and growth of type II and type I/III Mycobacterium avium subsp. paratuberculosis strains isolated from cattle and goats," Applied and Environmental Microbiology, vol. 72, no. 9, pp. 5927-5932, 2006.

[18] C. H. J. Kalis, M. T. Collins, J. W. Hesselink, and H. W. Barkema, "Specificity of two tests for the early diagnosis of bovine paratuberculosis based on cell-mediated immunity: the Johnin skin test and the gamma interferon assay," Veterinary Microbiology, vol. 97, no. 1-2, pp. 73-86, 2003.

[19] M. C. Antognoli, H. L. Hirst, F. B. Garry, and M. D. Salman, "Immune response to and faecal shedding of Mycobacterium avium ssp. paratuberculosis in young dairy calves, and the association between test results in the calves and the infection status of their dams," Zoonoses and Public Health, vol. 54, no. 3-4, pp. 152-159, 2007.

[20] F. Bastida and R. A. Juste, "Paratuberculosis control: a review with a focus on vaccination," Journal of Immune Based Therapies and Vaccines, vol. 9, no. 8, pp. 1-17, 2011.

[21] M. T. Collins, S. J. Wells, K. R. Petrini, J. E. Collins, R. D. Schultz, and R. H. Whitlock, "Evaluation of five antibody detection tests for diagnosis of bovine paratuberculosis," Clinical and Diagnostic Laboratory Immunology, vol. 12, no. 6, pp. 685692, 2005.

[22] S. S. Nielsen and N. Toft, "Ante mortem diagnosis of paratuberculosis: a review of accuracies of ELISA, interferon- $\gamma$ assay and faecal culture techniques," Veterinary Microbiology, vol. 129, no. 3-4, pp. 217-235, 2008.

[23] L. R. Gilardoni, F. A. Paolicchi, and S. L. Mundo, "Bovine paratuberculosis: a review of advantages and disadvantages of the different diagnostic tests," Revista Argentina de Microbiología, vol. 44, no. 3, 2012.

[24] A. P. Koets, V. P. M. G. Rutten, M. de Boer, D. Bakker, P. Valentin-Weigand, and W. van Eden, "Differential changes in heat shock protein-, lipoarabinomannan-, and purified protein derivative-specific immunoglobulin G1 and G2 isotype responses during bovine Mycobacterium avium subsp. paratuberculosis infection," Infection and Immunity, vol. 69, no. 3, pp. 1492-1498, 2001.

[25] B. Fernández, A. Jolly, S. Colavecchia, E. Fernández, and S. L. Mundo, "Efecto de la infección de Mycobacterium avium subsp. paratuberculosis en la producción de anticuerpos bovinos," Investigación Veterinaria, vol. 13, no. 1, pp. 9-17, 2011.

[26] D. M. Estes and W. C. Brown, "Type 1 and type 2 responses in regulation of Ig isotype expression in cattle," Veterinary Immunology and Immunopathology, vol. 90, no. 1-2, pp. 1-10, 2002.
[27] S. L. Mundo, "Respuesta Inmune en bovinos frente a Mycobacterium avium subsp. paratuberculosis," Universidad de Buenos Aires, Buenos Aires Argentina, 2005.

[28] L. Gilardoni, B. Fernández, A. M. Jar, C. Morsella, K. Cirone et al., "Mycobacterum avium paratuberculosis capture from milk using monoclonal and polyclonal antibodies linked to immunomagnetic beads," Reunión de La Sociedad Latinoamericana de Tuberculosis y otras Micobacterias, Rosario, Argentina, 2009.

[29] Y. Yokomizo, H. Yugi, and R. S. Merkal, "A method for avoiding false-positive reactions in an enzyme-linked immunosorbent assay (ELISA) for the diagnosis of bovine paratuberculosis," Nippon Juigaku Zasshi, vol. 47, no. 1, pp. 111-119, 1985.

[30] M. Greiner, D. Pfeiffer, and R. D. Smith, "Principles and practical application of the receiver-operating characteristic analysis for diagnostic tests," Preventive Veterinary Medicine, vol. 45, no. 1-2, pp. 23-41, 2000.

[31] A. Huda, G. Jungersen, and P. Lind, "Longitudinal study of interferon- $\gamma$, serum antibody and milk antibody responses in cattle infected with Mycobacterium avium subsp. paratuberculosis," Veterinary Microbiology, vol. 104, no. 1-2, pp. 43-53, 2004.

[32] E. R. DeLong, D. M. DeLong, and D. L. Clarke-Pearson, "Comparing the areas under two or more correlated receiver operating characteristic curves: a nonparametric approach," Biometrics, vol. 44, no. 3, pp. 837-845, 1988.

[33] F. A. Paolicchi, M. J. Zumárraga, A. Gioffré et al., "Application of different methods for the diagnosis of paratuberculosis in a dairy cattle herd in Argentina," Journal of Veterinary Medicine, Series B, vol. 50, no. 1, pp. 20-26, 2003.

[34] S. Eda, J. P. Bannantine, W. R. Waters et al., "A highly sensitive and subspecies-specific surface antigen enzyme-linked immunosorbent assay for diagnosis of Johne's disease," Clinical and Vaccine Immunology, vol. 13, no. 8, pp. 837-844, 2006.

[35] M. E. Fecteau and R. H. Whitlock, "Paratuberculosis in cattle," in Paratuberculosis: Organism, Disease, Control, M. A. Behr and D. M. Collins, Eds., pp. 144-156, CAB International 2010, 2010, section 14.

[36] M. C. Scott, J. P. Bannantine, Y. Kaneko et al., "Absorbed EVELISA: a diagnostic test with improved specificity for Johne's disease in cattle," Foodborne Pathogens and Disease, vol. 7, no. 11, pp. 1291-1296, 2010.

[37] S. S. Nielsen, "Immune-based diagnosis of paratuberculosis," in Paratuberculosis: Organism, Disease, Control, M. A. Behr and D. M. Collins, Eds., pp. 284-293, CAB International 2010, 2010, section 24 .

[38] C. A. Speer, M. C. Scott, J. P. Bannantine et al., "A novel enzyme-linked immunosorbent assay for diagnosis of Mycobacterium avium subsp. paratuberculosis infections (Johne's disease) in cattle," Clinical and Vaccine Immunology, vol. 13, no. 5, pp. 535-540, 2006. 

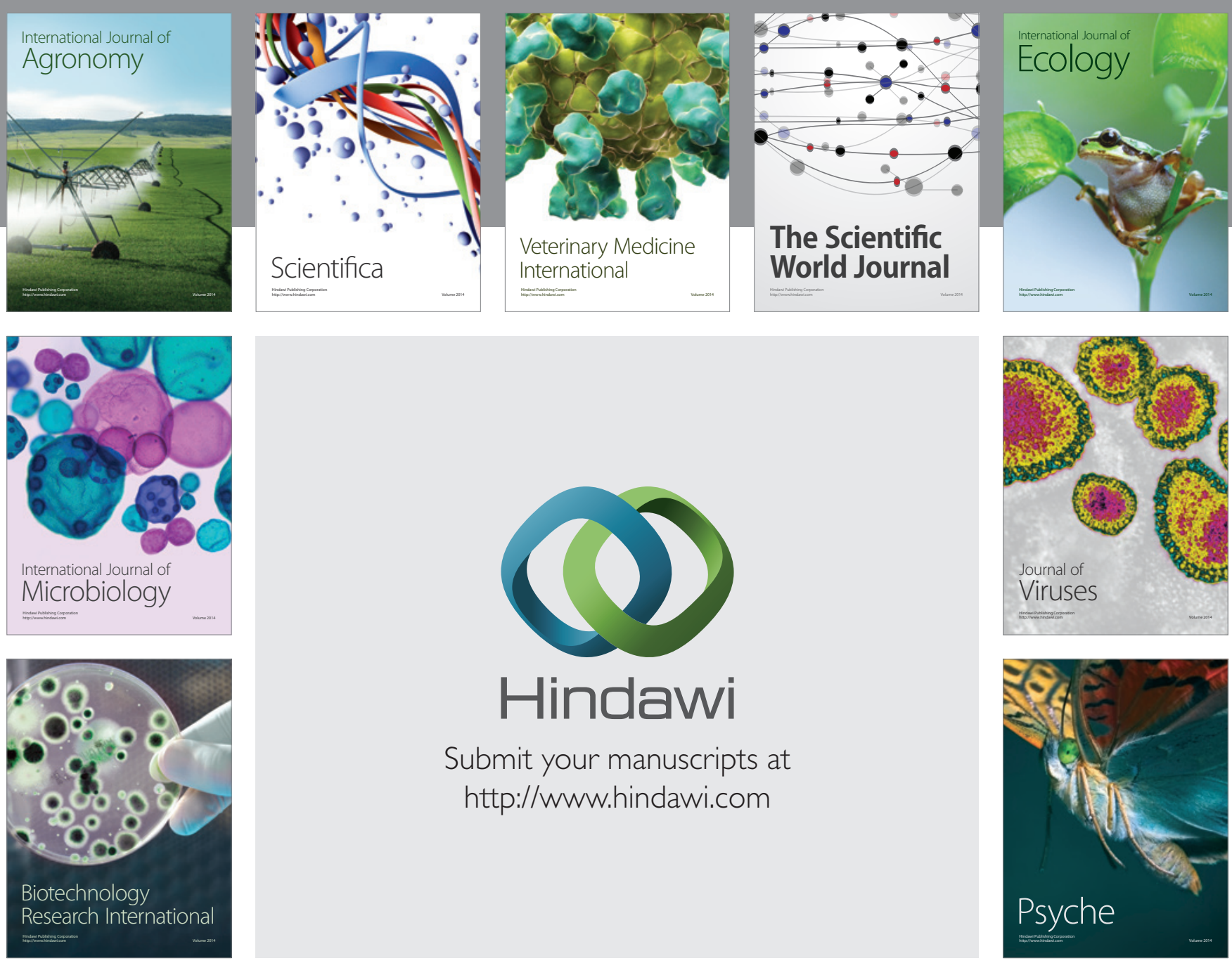

Submit your manuscripts at

http://www.hindawi.com
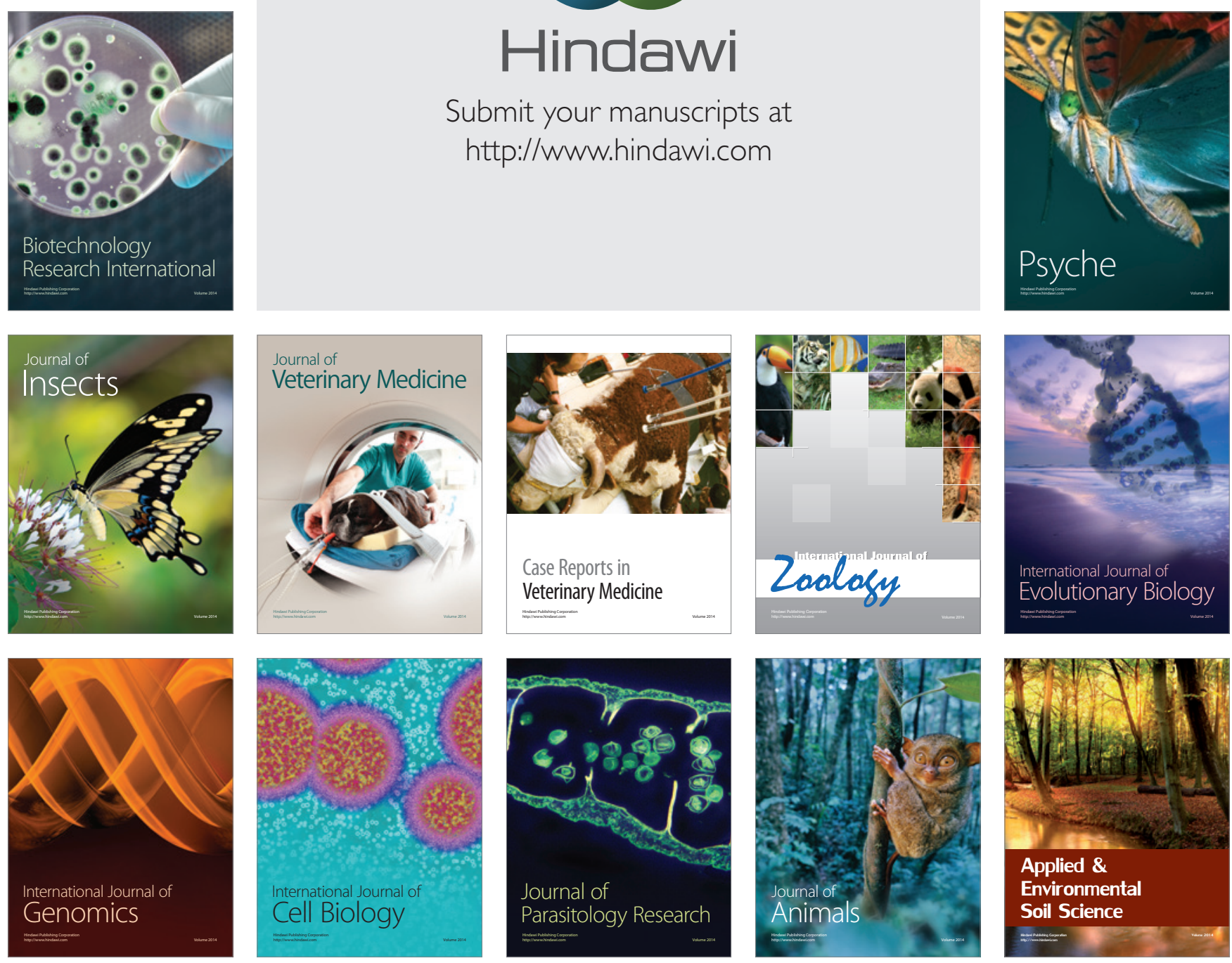